\title{
Retrospective study of the course, treatment and long-term follow-up of Kawasaki disease: a single-center experience from Poland
}

\author{
Aleksandra Stasiak $^{1}$ (D) Elżbieta Smolewska ${ }^{1}$ (D)
}

Received: 8 February 2019 / Accepted: 13 March 2019 / Published online: 22 March 2019

(c) The Author(s) 2019

\begin{abstract}
Kawasaki disease (KD) is an acute, self-limited, systemic vasculitis and the most common cause of acquired coronary artery disease in pediatric population in the developed countries. It occurs mostly in Asian countries; however, due to better access to diagnostic and imaging tests, it is more frequently diagnosed among pediatric patients in Poland. The aim of this study was to describe the clinical course with special interest in cardiac involvement, treatment and follow-up of Polish patients with KD. It is a single-center retrospective study. Clinical features (including coronary involvement), laboratory results and treatment were evaluated. In our study group, we observed elevated levels of indicators of inflammation: erythrocyte sedimentation rate (ESR), C-reactive protein (CRP), leukocytosis, platelet count, fibrinogen, D-dimer and ferritin. We also noticed changes in lipid profile and liver enzymes. Twenty-four patients were diagnosed with coronary artery abnormalities. Mean day of treatment equaled 9th day of the disease. Kawasaki disease should be suspected in all pediatric patients who have fever lasting 5 days, or more particularly those under 5 years of age. It is very important to apply treatment within the first 10 days of disease due to the high risk of cardiovascular complications. Each child should have echocardiography on admission, around 14th day of the disease, after 4-6 weeks from the onset of symptoms, as well as long-term observation at least once a year due to the fact that the inflammatory process and changes in the lipid profile increase the risk of atherosclerosis. Children with coronary aneurysms should undergo check-ups every 6 months.
\end{abstract}

Keywords Kawasaki disease $\cdot$ Children · Diagnosis · Vasculitis

\section{Introduction}

Kawasaki disease (KD) is defined as an acute, self-limited, systemic vasculitis. It is mostly observed in children between 6 months and 5 years of age with prevalence to Asian population. $\mathrm{KD}$ is the most common cause of acquired coronary artery disease in pediatric population in the developed countries [1]. The disease was first observed and described in 1967 as an acute mucocutaneous lymph node syndrome affecting the skin, mucosa and lymph nodes [2]. Currently, $\mathrm{KD}$ is defined as the presence of a fever lasting $\geq 5$ days, accompanied by four out of five of the following symptoms:

Elżbieta Smolewska

e.smolewska@wp.pl

Aleksandra Stasiak

aleksandra.stasiak12@gmail.com

1 Department of Pediatric Cardiology and Rheumatology, Medical University of Lodz, Sporna 36/50 street, 91-738 Lodz, Poland polymorphous rash, bilateral conjunctival injection, oral changes such as cracked and erythematous lips and strawberry tongue, cervical lymphadenopathy, extremity changes such as erythema or palm and sole desquamation [1]. Incomplete KD includes fever lasting $\geq 5$ days and two or three of the symptoms stated above. The term "atypical Kawasaki disease" is used to describe children who fail to meet the classic KD criteria but have compatible laboratory findings and no other explanation for their illness [2]. Some authors believe that this term should be reserved for patients who have a problem that generally is not seen in KD, such as renal impairment, arthritis, aseptic meningitis, pneumonitis, uveitis, gastroenteritis or otitis $[3,4]$.

There are still no specific laboratory tests pathognomonic for the $\mathrm{KD}$; however, clinical experience suggests that a C-reactive protein $(\mathrm{CRP}) \geq 3.0 \mathrm{mg} / \mathrm{dl}$ and/or erythrocyte sedimentation rate (ESR) $\geq 40 \mathrm{~mm} / \mathrm{hr}$ are supportive of KD. Patients with these laboratory findings and a positive echocardiogram, or $\geq 3$ supportive laboratory findings (anemia, platelet count (PLT) of $\geq 450 \mathrm{~g} / \mathrm{l}$ after the seventh day 
of fever, albumin $\leq 3.0 \mathrm{~g} / \mathrm{dl}$, elevated alanine aminotransferase (ALT), white blood cell (WBC) count $\geq 15.0 \mathrm{~g} / \mathrm{l}$ ) should be diagnosed with KD [1]. Echocardiography has a high sensitivity and specificity for the detection of abnormalities of the proximal coronary artery segments [1]. About $20 \%$ of patients develop coronary artery aneurysms without proper treatment in a timely manner during the acute phase of coronary artery complications [5]. Treatment of the KD includes an intravenous immunoglobulin (IVIG) infusion. Administration of moderate to high dose of acetylsalicylic acid (ASA) should be continued until the patient is afebrile [1].

Several attempts have been made to develop a gold standard for diagnostic test in KD. The aim of this study was to describe the clinical course with special interest in cardiac involvement, treatment and follow-up of Polish patients with $\mathrm{KD}$ as an experience of a single-center study.

\section{Materials and methods}

The study has been performed to describe the characteristics of a cohort of KD patients based on the information acquired from database of patients hospitalized in the single Polish center in years 2010-2018. The study has been performed according to the standard Helsinki Declaration.

Diagnosis was based on clinical criteria described in American Heart Association (AHA) scientific statement on diagnosis, treatment, and long-term management of KD. Clinical features, laboratory results and treatment were evaluated. Besides medical history and careful physical examination, functional and imaging examinations, such as transthoracic echocardiography, electrocardiogram (ECG), and chest X-ray (CXR) examinations were assessed in each child.

Several laboratory tests were taken into consideration as supportive of the KD diagnosis. According to the AHA guidelines, ALT activity and albumin levels were also taken into consideration in this study. Apart from these guidelines, we also assessed some other parameters that were tested in previous studies of KD to prepare an algorithm for clinical aid of the diagnosis. These included peripheral complete blood count, gamma-glutamyl transferase (GGTP), fibrinogen, D-dimers or ferritin serum levels, as well as the full lipoprotein profile. All those laboratory tests were done by the standard methods.

\section{Statistical analyses}

For continuous variables, the median, maximal and minimal values were calculated. All values are presented as median (minimum; maximum). Categorical variables are presented as fractions. To investigate the correlations between the variables Spearman's rank correlation was applied. A $p$ value $\leq 0.05$ was considered to be statistically significant. Kolmogorov-Smirnov test was utilized to check the normality of distribution of continuous variables. Tested parameters showed non-normal distribution in the Kolmogorov-Smirnov test $(p \geq 0.005)$. Statistical analysis was performed using the Dell Statistica 13 data analysis software (StatSoft Polska, Krakow, Poland).

\section{Results}

\section{Clinical characteristics}

The study group consisted of 37 patients ( 24 boys and 13 girls). The median age of the patients was 3 years, (min. 0.4; max. 10 years). The median time of hospitalization equaled 14 days (min. 6; max. 32 days).

Sixteen out of thirty-seven children were admitted to our Department from another hospital with suspicion of pneumonia, and three patients with a diagnosis of pharyngitis. One patient (1/37) was previously treated for Herpes simplex virus (HSV) infection, four patients (4/37) were admitted as unknown infection, and another two children (2/37) were initially treated for sepsis/neuroinfection. Two patients (2/37) had lymphadenopathy and one patient (1/37) had gastrointestinal infection. One child (1/37) had a suspicion of connective tissue disease. Only 7 out of 37 patients were referred to a hospital on suspicion of KD (Table 1).

All the patients had fever present for at least 5 days; in three-fourth of the cases, this symptom was due to KD. Nine out of thirty-seven children were diagnosed as incomplete KD. Other symptoms commonly associated with the typical indicators of KD were hepatomegaly (13/37 patients), arthritis (9/37 patients), hyperesthesia (5/37 patients), and splenomegaly (one patient) (Fig. 1). Only one child was diagnosed

Table 1 Initial diagnosis of patients in the study group prior to admission to the Department of Pediatric Cardiology and Rheumatology

\begin{tabular}{ll}
\hline Diagnosis & $\begin{array}{l}\text { Number } \\
\text { of chil- } \\
\text { dren }\end{array}$ \\
\hline Pneumonia & $16 / 37$ \\
Pharyngitis & $3 / 37$ \\
Sepsis/neuroinfection & $2 / 37$ \\
Lymphadenitis & $2 / 37$ \\
HSV infection & $1 / 37$ \\
Gastrointestinal infection & $1 / 37$ \\
Connective tissue disease & $1 / 37$ \\
Unknown infection & $4 / 37$ \\
Suspicion of Kawasaki disease & $7 / 37$ \\
\hline
\end{tabular}


Fig. 1 Symptoms associated with $\mathrm{KD}$ presented by the study group

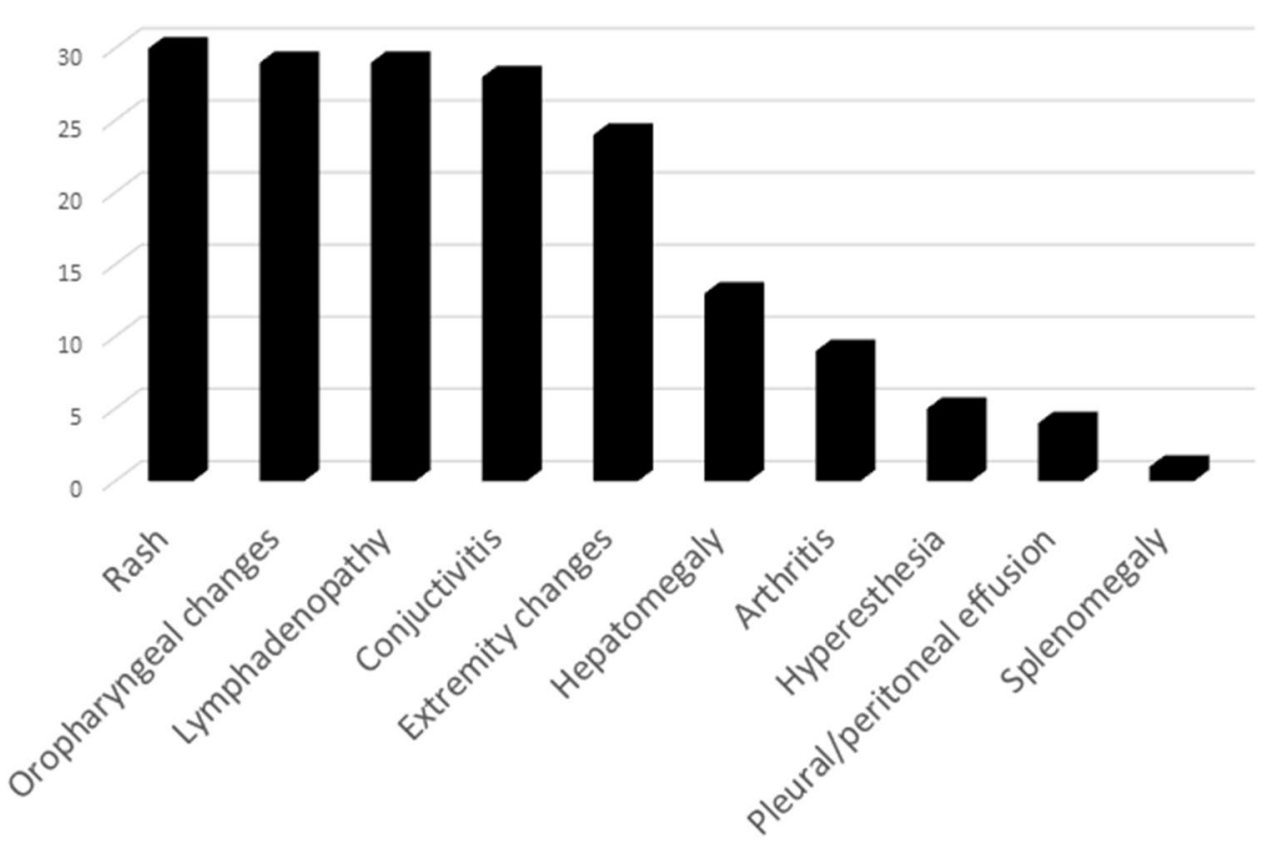

with atypical KD; he presented fever and three criteria of KD; however, unusual symptoms such as arthritis, hyperesthesia, hepatomegaly and peritoneal effusion occurred.

Twenty-nine out of thirty-seven patients had oropharyngeal changes such as cracked or erythematous lips or strawberry tongue. In three-fourth of children, lymphadenopathy and conjunctivitis were detected.

Additionally, 30 children (30/37) had a skin polymorphous rash. Twenty-four children (24/37) had erythema of palms and sole desquamation at the time of admission or during the course of treatment.

In the whole group, 32 children had a correct resting ECG, 2 children in the study group had repolarization abnormalities and three children had intraventricular conduction changes.

The least detected symptom in the study group was pleural or peritoneal effusion, which occurred in four children (4/37; one case of pleural effusion and three cases of peritoneal effusion), respectively.

\section{Laboratory tests}

The median ALT and albumin levels were 24.0 (6.0; $337.0) \mathrm{U} / \mathrm{l}$ and $3.7(2.9 ; 8.2) \mathrm{mg} / \mathrm{dl}$, respectively (Table 2).

Among other laboratory tests the median GGTP level was 47.0 (11.0; 314.0) U/l and hemoglobin level was 10.8 (8.1; $15.3) \mathrm{g} / \mathrm{dl}$. A median WBC count was $13.3(5.9 ; 52.6) \mathrm{g} / \mathrm{l}$, with median monocytes value of $7.0(3.0 ; 24.0) \mathrm{g} / \mathrm{l}$, eosinophils $3.0(1.0 ; 20.0) \mathrm{g} / \mathrm{l}$, and immature neutrophil percentage count of 6.0 (6-22) \%. Median PLT count was 704.0 (130.0; 1.100.0) $\mathrm{g} / \mathrm{l}$. ESR in the study group equaled 95.0 (28.0;
133) $\mathrm{mm} / \mathrm{hr}$. Median CRP level in children with KD was $32.9(0.1 ; 221) \mathrm{mg} / \mathrm{dl}$.

Additionally, median ferritin level was 209.9 (85.3; 6816.0) $\mu \mathrm{g} / \mathrm{l}$, a median serum fibrinogen level 519.0 (259.4; $704.5) \mathrm{mg} / \mathrm{dl}$, and a median D-dimer level was 1002.8 (393.0; 8618.0) ng/ml. Lipid profile checked prior to treatment and results were for serum cholesterol level median value of $147.5(70 ; 242) \mathrm{mg} / \mathrm{dl}$, high-density lipoprotein (HDL) $25.2(3.4 ; 43.0) \mathrm{g} / \mathrm{dl}$, low-density lipoprotein (LDL) $95.0(6.7 ; 203.0) \mathrm{mg} / \mathrm{dl}$, and triglyceride level 144.0 (54; 318.0) $\mathrm{mg} / \mathrm{dl}$ (Table 2).

\section{Treatment}

The applied treatment included an infusion of IVIG at a dose of $2 \mathrm{~g} / \mathrm{kg}$, an oral dose of ASA initially in an antiinflammatory dose, then in an anticoagulant dose. Two patients (2/37) received only ASA therapy due to the mild course of the disease and no changes in the coronary arteries. Four patients (4/37) required a double infusion of IVIG. In seven patients (7/37), systemic steroid therapy was additionally applied, mostly in patients with persistent arthritis and fever. One patient required an intravenous infusion of albumins. Mean day of administration of IVIG in the study group was between 9th and 10th day of the disease. Eleven out of thirty-seven patients received IVIG treatment after the 10th day of the disease. All of these patients presented abnormalities in the echocardiogram: three patients had vulvar regurgitations, five patients had dilatation of coronary arteries, eight patients suffered from pericardial effusion and two patients developed coronary artery aneurysms (some patients presented more than one abnormality). 
Table 2 Laboratory findings during acute phase of $\mathrm{KD}$ in the study group

\begin{tabular}{llcll}
\hline Laboratory test & Unit (SI) & Median & Min; max & Norm \\
\hline ESR & $\mathrm{mm} / 1 \mathrm{~h}$ & 95.0 & $28.0 ; 133.0$ & $0-10$ \\
$\mathrm{CRP}$ & $\mathrm{mg} / \mathrm{dl}$ & 32.9 & $0.1 ; 221.0$ & $0-5.0$ \\
$\mathrm{PLT}$ & $\mathrm{g} / \mathrm{l}$ & 704.0 & $130.0 ; 1.1000 .0$ & $150.0-400.0$ \\
$\mathrm{ALT}$ & $\mathrm{U} / \mathrm{l}$ & 24.0 & $6.0 ; 337.0$ & $0-41$ \\
Albumin & $\mathrm{g} / \mathrm{dl}$ & 3.7 & $2.9 ; 8.2$ & $3.8-5.4$ \\
HGB & $\mathrm{g} / \mathrm{dl}$ & 10.7 & $8.1 ; 15.3$ & $11.0-13.8$ \\
WBC & $\mathrm{g} / \mathrm{l}$ & 13.3 & $5.9 ; 52.6$ & $2.5-13.0$ \\
MONO & $\mathrm{g} / \mathrm{l}$ & 7.0 & $3.0 ; 24.0$ & $0.2-1.3$ \\
EO & $\mathrm{g} / \mathrm{l}$ & 3.0 & $1.0 ; 20.0$ & $0.1-1.2$ \\
Immature neutrophils & $\%$ (fraction & 6 & $1 ; 22$ & $3-6$ \\
GGTP & $\mathrm{U} / \mathrm{l}$ & 47.0 & $11.0 ; 314.0$ & $0-18.0$ \\
Fibrinogen & $\mathrm{mg} / \mathrm{dl}$ & 519.0 & $259.4 ; 740.5$ & $200.0-400.0$ \\
D-dimer & $\mathrm{ng} / \mathrm{ml}$ & 1002.8 & $393.0 ; 8618.0$ & $0.0-500.0$ \\
Ferritin & $\mu \mathrm{g} / \mathrm{l}$ & 209.9 & $85.3 ; 6816.0$ & $6.0-67.0$ \\
Cholesterol & $\mathrm{mg} / \mathrm{dl}$ & 147.5 & $70.0 ; 242.0$ & $<170$ \\
HDL & $\mathrm{mg} / \mathrm{dl}$ & 25.2 & $3.4 ; 43.0$ & $>40$ \\
LDL & $\mathrm{mg} / \mathrm{dl}$ & 95.0 & $6.7 ; 203.0$ & $<110$ \\
Triglycerides & $\mathrm{mg} / \mathrm{dl}$ & 144.0 & $54.0 ; 318.0$ & $<150$ \\
\hline
\end{tabular}

Norm - a correct value of a given laboratory parameter according to the laboratory where the test was performed

ESR erythrocyte sedimentation rate, $C R P$ C-reactive protein, $P L T$ platelets, $H G B$ hemoglobin, $W B C$ white blood cells, $A L T$ alanine aminotransferase, GGTP gamma glutamyl transpeptidase, $E O$ eosinophils, $M O N O$ monocytes
Each patient had a follow-up 4 weeks after the diagnosis. Thirty-five out of thirty-seven children obtained normalization of CRP level and thirty-one out of thirty-seven children obtained a correct ESR. Ten patients (10/37) still had elevated PLT count at the 4-week follow-up. Eighteen of patients (18/37) required maintenance of anticoagulant therapy in a form of acetylsalicylic acid due to sustained changes in coronary arteries. After 4 weeks, changes in the lipid profile of the children were at the same level as during the acute phase of the disease. One child had elevated fibrinogen level and two children had elevated D-dimer level after 4 weeks.

Twenty-two out of thirty-seven patients needed another check-up after the next 12 weeks. Eight of them still required anticoagulant therapy due to persistent widening of coronary arteries.

The hemoglobin level correlated inversely with the PLT count $(p \leq 0.001)$ and ESR $(p \leq 0.0001)$. ESR positively correlated with PLT count $(p \leq 0.002)$, but not with CRP level. Higher PLT count correlated positively with ESR $(p \leq 0.002)$ and WBC count $(p \leq 0.0004)$. Higher WBC count correlated with higher levels of CRP $(p \leq 0.003)$, PLT count $(p \leq 0.0004)$ and fibrinogen $(p \leq 0.0008)$, but not with ESR. ALT levels correlated positively with GGTP $(p \leq 0.0001)$. There was no statistically significant correlation between any of the laboratory parameters and the age of children, days of hospitalization or presence of abnormalities in coronary arteries.

\section{Discussion}

Despite the fact that KD is an illness known for over 50 years, the pathogenesis of this disease as well as a pathognomonic test enabling rapid diagnosis were still not determined. According to AHA incident rate of the KD in The USA equals 25 per 100,000 hospitalized children with prevalence of Asian ethnicity [1]. The incidence of KD differs widely among ethnic groups and is higher in the Asian populations (in Japan 239.6/100,000 children $<5$ years of age per year) than in Europe, where the incidence ranges from 4.9 to $15.2 / 100,000 /$ year in the same age group [6].

Epidemiological data for KD in Poland are still unknown. Recurrence rate of the KD is estimated to be about $3 \%$ in Japan. Fatality record of the KD in Japan is $0.015 \%$. KD is more common in boys, who outnumber girls by $\approx 1.5-1.7: 1$, and $76 \%$ of affected children is under the age of 5 [1]. In our study group, we obtained similar results as boy to girl ratio equaled $1.8: 1$, and $81 \%$ of the children were under the age of 5 .

Recent reports showed steady increase in the prevalence of KD. Viral or bacterial infections, autoimmune factors, 
and genetic factors have been suggested as a key agents in KD pathogenesis [7, 8]. Our study confirms these observations, at least in regard to prevalence of infections preceding KD diagnosis. Importantly, all our patients had fever lasting 5 or more days. This may reflect activity of proinflammatory cytokines, believed to mediate the underlying coronary artery inflammation.

Fig. 2 Abnormalities found in echocardiography

Fig. 3 Aneurysms of the right and left coronary arteries in an 8-month-old patient. 1-Right coronary artery aneurysm, 2 aorta, 3-left coronary artery aneurysm
Among our patients, nearly $1 / 4$ of cases met the criteria of incomplete KD. Thirty-six out of thirty-seven patients presented abnormalities in echocardiogram (Figs. 2, 3). Among 24 patients with dilatations/aneurysms of coronary arteries, six patients (1/4 of the group) presented incomplete KD and five patients (1/5 of the group) received IVIG treatment after the 10th day of disease. This group of patients presented
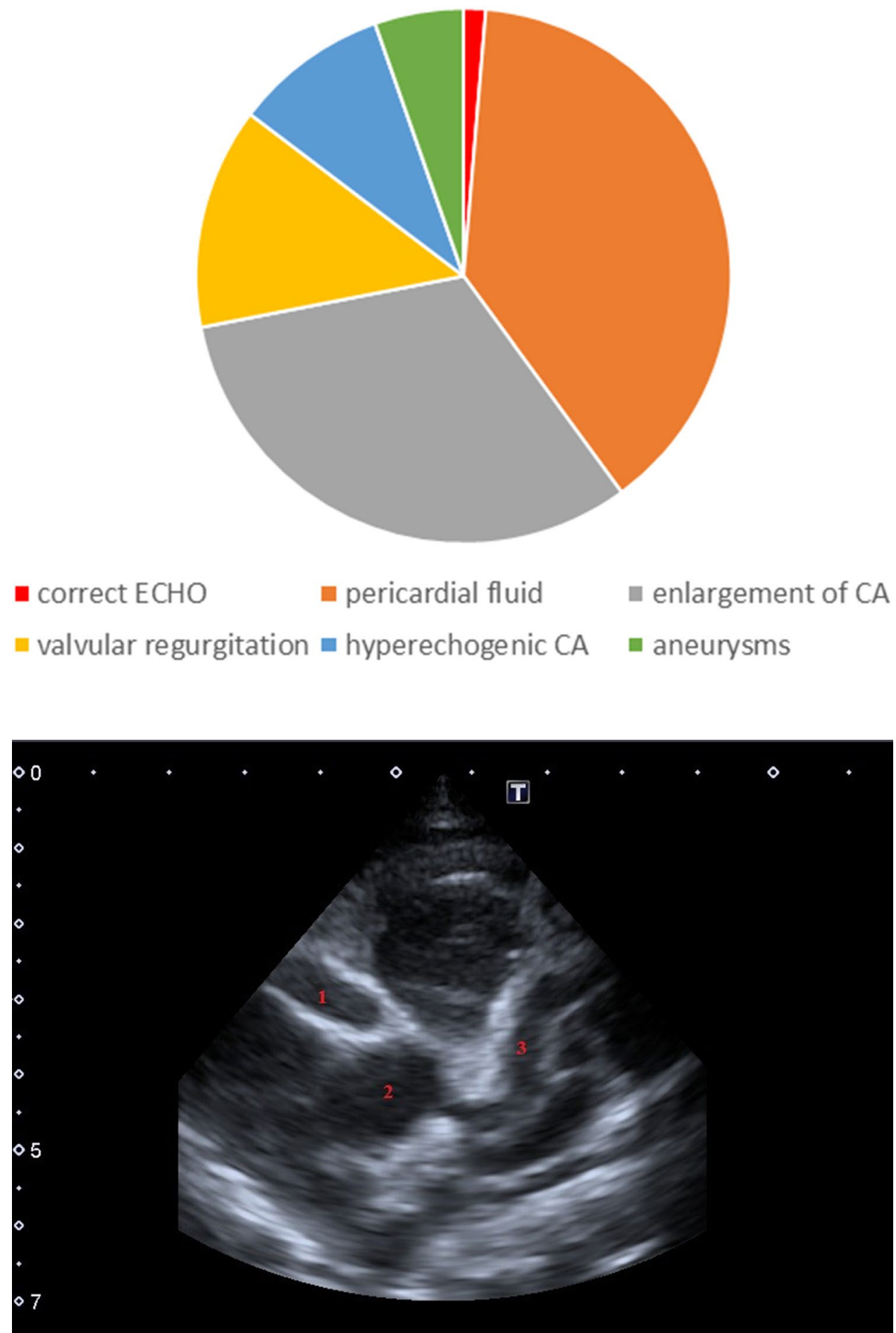
higher ESR level and higher platelet count than a group of patients without changes in the coronary arteries. In addition, $86.6 \%$ of this group was male, and the median age of patients was 1.9 years, which is consistent with other reports which state that male gender and younger age are a risk factor for the development of coronary artery abnormalities [9]. There was no statistically significant correlation between coronary artery aneurysms and results of laboratory tests. Mean day of administration of IVIG in the study group was between 9th and 10th day of the disease. Such a delayed time of IVIG administration was caused by an extended period of time after which some patients were admitted to our department from local hospitals. Due to persistent inflammation and confirmation of the diagnosis, we decided to administer IVIG treatment despite the fact that illness lasted 10 days or more. Two children in this group required second administration of IVIG and another two patients received glucocorticosteroids as additional treatment. In our study, almost half of the patients still required ASA treatment at 6-week follow-up due to persistent changes in the cardiovascular system.

KD should be suspected in all children below the age of 5 , with persistent fever unresponsive to antipyretic and antibiotic therapy, especially if it is accompanied by symptoms such as rash, conjunctivitis, extremity changes, oropharyngeal changes or cervical lymphadenopathy. These children should have appropriate laboratory tests to confirm KD diagnosis and should be consulted by a cardiologist with echocardiogram performed as soon as possible. If diagnosis is unsure, serial clinical and laboratory re-evaluations should be performed. IVIG treatment should be applied within 10 days of illness onset but as soon as possible after diagnosis [1].

Diagnosis is based on clinical features. In our study, $3 / 4$ of the patients presented bilateral conjunctivitis, as well as oropharyngeal changes, including cracked or erythematous lips or strawberry tongue. Majority of the study group presented extremity changes such as erythema or palm and sole desquamation, which was consistent with other reports [1]. Although nonspecific and nondiagnostic lymphadenopathy occurred in $3 / 4$ of our patients and mainly regarded the anterior cervical nodes, as stated in other reports, this symptom tends to involve primarily the anterior cervical nodes overlying the sternocleidomastoid muscle [1].

It is known that in its active phase, $\mathrm{KD}$ is accompanied by systemic inflammation in all medium-sized arteries and in multiple other tissues leading to hepatitis, pericarditis or pneumonitis [1]. Other clinical findings may include pleu$\mathrm{ral} /$ peritoneal effusion, which occurred in four patients, and arthritis, which occurred in nearly $1 / 4$ of cases in our study, and included small joints of hands and feet, knees and hips. Small joint polyarthritis during the first week of illness, followed by a large joint arthritis, mainly involving lower extremities occurs, according to Canadian population, in approximately $7.5 \%$ of $\mathrm{KD}[8,10]$.

Laboratory tests, although nonspecific, provide support for a diagnosis of KD [1]. Systemic inflammation is most characteristic, manifested by increase of acute phase reactants, leukocytosis, and a left shift in the white blood cell count [8]. Ling et al. measured KD data, which included 12 variables: total WBC count, percentages of monocytes, lymphocytes, eosinophils, neutrophils, and immature neutrophils, PLT count, hemoglobin concentration, CRP, GGTP, ALT, and ESR [11]. In our study group, we found elevated levels of ESR, CRP, leukocytosis and neutrophil count. Importantly, ESR was elevated by IVIG therapy and, therefore, a decreased ESR during follow-up is not a marker of a response to IVIG treatment. Almost all children had normalization of CRP and correction in ESR in most cases after 4 weeks from the onset of therapy. Mean highest PLT count in our study occurred after 7th day of fever in most cases and in around $1 / 4$ of the group this elevation persisted at the 4-week follow-up.

Twenty-two children had lipid profile checked prior to treatment. Iranian and Indian studies suggest that children who went through KD are more prone to changes in their lipid profile, especially in the level of triglycerides and HDL, which may lead to premature atherosclerosis [12, 13]. Periodic control of lipid values in these patients is of crucial importance [12]. In our study group, we noticed changes in the mean HDL and triglycerides levels. Almost every patient had a lipid profile checked 4 weeks after the treatment.

Cardiovascular manifestations during KD episode include coronary artery lesions and can lead to long-term morbidity and mortality [14]. Pericardium, myocardium, endocardium including valves, and coronary arteries may all be inflamed [1]. Transthoracic echocardiography is the diagnostic method of choice to screen for changes or aneurysms in coronary arteries and should be obtained within the first 10 days of illness [15]. In our group, only one patient presented correct anatomy and function of the heart. Coronary aneurysms or dilatations resulting from $\mathrm{KD}$ are known to change over time, sometimes with regression [5]. Without early treatment, about $20 \%$ of patients develop coronary artery aneurysms, leading to ischemic heart disease, myocardial infarction, or even sudden death [14]. Echocardiography should be repeated both within 1-2 weeks and 4-6 weeks after treatment [1]. Majority of children had a correct resting ECG; repolarization abnormalities and intraventricular conduction disorders were recorded in 5/37 children. None of the children developed arrhythmia.

In comparison with a retrospective study conducted in a single Irish tertiary center during 4-year period treatment, coronary artery outcomes were examined in a cohort of 32 pediatric patients with KD. Forty-seven percent had abnormal echocardiogram findings in the 
acute phase. Twenty-five percent had echocardiogram abnormalities at 6 -week follow-up. Twenty-eight percent of patients had an incomplete diagnosis of KD [16]. In a study conducted in two Italian regions during a 6-year period, a total of 426 first hospital admissions for KD were recorded. Five percent of patients had at least one cardiac complication [6]. We diagnosed presence of pericardial fluid in 29/37 of patients, enlargement of the coronary arteries in almost $24 / 37$ of patients and in $4 / 37$ patients coronary artery aneurysms. In ten of our cases mitral and aortic valve regurgitation was observed. A retrospective study of 27 patients with KD hospitalized at three pediatric rheumatology centers in Poland showed that the small coronary artery aneurysms were noted in $25.9 \%$ of patients [17]. Each patient had a cardiological assessment 6 months after discharge from the hospital. In our center each child receives a check-up about 1-2 weeks from the onset of disease, as well as after 4-6 weeks and if necessary after 12 weeks. Additionally, all children undergo a full cardiological assessment at least once a year.

Treatment should be applied as fast as possible to reduce inflammation and prevent changes in coronary arteries as well as possible thrombosis [1]. Patients should be treated with IVIG $2 \mathrm{~g} / \mathrm{kg}$ as a single infusion, usually given over 10-12 h, along with ASA. If there is no response to treatment, patients are given a second dose of IVIG with or without corticosteroids or other adjunctive treatment [15]. Administration of glucocorticosteroids should be reserved for patients with high risk of coronary aneurysms [1]. Administration of IVIG has been shown to reduce the incidence of coronary aneurysms significantly to $2-3 \%$ if given within the first 10 days [5]. In our study, IVIG was administered at a dose of $2 \mathrm{~g} / \mathrm{kg}$, along with an oral dose of ASA initially in an anti-inflammatory dose $(80-100 \mathrm{mg} / \mathrm{kg}$ ), then in an anticoagulant dose. Two out of thirty-seven patients received only ASA therapy due to the mild course of the disease and no changes in the coronary arteries, whereas approximately four of patients required a double infusion of IVIG. In 1/5 of the patients, systemic glucocorticosteroid therapy was additionally applied.

Recent evidence suggests that treatment with corticosteroids added to IVIG/ASA therapy reduces complications in high-risk KD [16]. Li et al. showed that the initial administration of IVIG $\leq 4.0$ days after the onset of symptoms, increased ESR and decreased hemoglobin and PLT counts, oral mucosa alterations, cervical lymphadenopathy, swelling of the extremities, and polymorphous rash are the risk factors for IVIG-resistant KD [18]. Finally, D-dimer elevation and albumin decline in IVIG-unresponsive KD patients could be risk factors for development of acute coronary artery lesions [19].

\section{Strengths and limitations}

It is worth emphasizing that the presented study is one of a few single-center KD studies in Poland. Correct identification of symptoms accompanying $\mathrm{KD}$, including rare symptoms such as body cavity exudates or arthritis is of crucial importance in the diagnostic process. Moreover to date, there are only few reports in the subject of lipid profile abnormalities in patients with Kawasaki disease, which can lead to premature formation of atherosclerotic plaques in the vessels. In our study, we noted abnormalities in lipid profile in children with $\mathrm{KD}$, which require more careful analysis and follow-up. The main limitations of the study were primarily a small group of patients, resulting from the region in which it was carried out (lower incidence rate), retrospective nature of the study and high percentage of cardiac complications.

\section{Conclusions}

Even though there is still no gold standard diagnostic test, Kawasaki disease should be suspected in all pediatric patients who have fever lasting 5 days, or more particularly those under 5 years of age. It is very important to apply treatment within the first 10 days of disease due to the high risk of cardiovascular complications. Each child should have echocardiography on admission, around 14th day of the disease, after 4-6 weeks from the onset of symptoms, as well as long-term observation at least once a year due to the fact that the inflammatory process and changes in the lipid profile increase the risk of atherosclerosis. Children with coronary aneurysms should undergo check-ups every 6 months.

Author contributions AS: literature search, study design, data collection, analysis, interpretation, figures, and writing. ES: study design, data analysis, interpretation, and writing.

Funding The study has been partially supported by the Grant from the Medical University of Lodz, Poland; Grant no. 503/8-000-04/503-81-002.

\section{Compliance with ethical standards}

Conflict of interest The authors declare that they have no conflict of interest.

Ethical approval Retrospective data was used for this study, ethics approval was not required for this study at our institution. All procedures were performed in accordance with ethical standards of the Helsinki declaration. Legal guardian of each patient consented to review of the medical records and to the use of the data. 
Open Access This article is distributed under the terms of the Creative Commons Attribution 4.0 International License (http://creativeco mmons.org/licenses/by/4.0/), which permits unrestricted use, distribution, and reproduction in any medium, provided you give appropriate credit to the original author(s) and the source, provide a link to the Creative Commons license, and indicate if changes were made.

\section{References}

1. McCrindle BW, Rowley AH, Newburger JW et al (2017) Diagnosis, treatment, and long-term management of Kawasaki disease: a scientific statement for health professionals from the American Heart Association. Circulation 135:e927-e999. https://doi. org/10.1161/CIR.0000000000000484

2. Barone SR, Pontrelli LR, Krilov LR (2000) The Differentiation of classic Kawasaki disease, atypical Kawasaki disease, and acute adenoviral infection: use of clinical features and a rapid direct fluorescent antigen test. Arch Pediatr Adolesc Med 154:453-456. https://doi.org/10.1001/archpedi.154.5.453

3. Yu JJ (2012) Diagnosis of incomplete Kawasaki disease. Korean J Pediatr 55:83-87

4. Hsieh YC, Wu MG, Wang JK et al (2002) Clinical features of atypical Kawasaki disease. J Microbiol Immunol Infect 35:60-67

5. Advani N, Sastroasmoro S, Ontoseno T, Uiterwaal CS (2018) Long-term outcome of coronary artery dilatation in Kawasaki disease. Ann Pediatr Cardiol 11:125-129. https://doi.org/10.4103/ apc.APC_172_16

6. Mauro A, Fabi M, Da Fre M, Gustarobo P, Corinaldesi E et al (2016) Kawasaki disease: epidemiological study in central Italy. Pediatr Rheumatol Online J 14:22. https://doi.org/10.1186/s1296 9-016-0084-6

7. Chang LY, Lu CY, Shao PL et al (2013) Viral infections associated with Kawasaki disease. J Formos Med Assoc 113:148-154. https ://doi.org/10.1016/j.jfma.2013.12.008

8. Sundel RP (2015) Kawasaki disease. Rheum Dis Clin North Am 41:63-73. https://doi.org/10.1016/j.rdc.2014.09.010

9. McCrindle BW, Li JS, Minich LL et al (2007) Coronary artery involvement in children with Kawasaki disease: risk factors from analysis of serial normalized measurements. Circulation 116:174 $-179$
10. Martins A, Conde M, Brito M, Gouveia C (2018) Arthritis in Kawasaki disease: a poorly recognised manifestation. J Paediatr Child Health 54:1371-1374. https://doi.org/10.1111/jpc.14102

11. Ling XB, Kanegaye JT, Ji J et al (2013) Point-of-care differentiation of Kawasaki disease from other febrile illnesses. J Pediatr 162:183-188. https://doi.org/10.1016/j.jpeds.2012.06.012

12. Mostafavi S-N, Barzegar E, Manssori NS, Kelishadi R (2014) First report on the lipid profile late after Kawasaki disease in Iranian children. Int J Prev Med 5:820-824

13. Narsaria P, Singh S, Gupta A, Khullar M, Bhalla A (2015) Lipid profile and fat patterning in children at a mean of 8.8 years after Kawasaki disease: a study from Northern India. Clin Exp Rheumatol 33:171-175

14. Kyu Yeun K, Dong Soo K (2016) Recent advances in Kawasaki disease. Yonsei Med J 57:15-21. https://doi.org/10.3349/ ymj.2016.57.1.15

15. Saguil A, Fargo M, Grogan S (2015) Diagnosis and management of Kawasaki disease. Am Fam Physician 91:365-371

16. Flinn AM, Gavin PJ, McMahon CJ, Oslizok P, Butler KM (2018) Kawasaki disease-a review of treatment and outcomes in an Irish Paediatric Cohort 2010-14. Ir Med J 111:691

17. Gorczyca D, Postępski J, Olesińska E et al (2013) The clinical profile of Kawasaki disease of children from three Polish centers: a retrospective study. Rheumatol Int 34:875-880

18. Li X, Chen Y, Tang Y et al (2018) Predictors of intravenous immunoglobulin-resistant Kawasaki disease in children: a metaanalysis of 4442 cases. Eur J Pediatr 177:1279-1292. https://doi. org/10.1007/s00431-018-3182-2

19. Masuzawa Y, Mori M, Hara T, Inaba A, Oba MS, Yokota S (2015) Elevated D-dimer level is a risk factor for coronary artery lesions accompanying intravenous immunoglobulin-unresponsive Kawasaki disease. Ther Apher Dial 19:171-177. https://doi. org/10.1111/1744-9987.12235

Publisher's Note Springer Nature remains neutral with regard to jurisdictional claims in published maps and institutional affiliations. 